\title{
Current Questions in HIV-Associated Lung Cancer
}

\author{
Marina Shcherba ${ }^{1}$, Jonathan Shuter ${ }^{2}$, and Missak Haigentz Jr. ${ }^{1}$ \\ ${ }^{1}$ Division of Oncology, Department of Medicine, Albert Einstein College of Medicine/Montefiore \\ Medical Center, Bronx, New York, USA \\ 2 Division of Infectious Diseases, Department of Medicine, Albert Einstein College of Medicine/ \\ Montefiore Medical Center, Bronx, New York, USA
}

\begin{abstract}
Purpose of review-In this review, we explore current questions regarding risk factors contributing to frequent and early onset of lung cancer among populations with HIV infection, treatment and outcomes of lung cancer in HIV-infected patients as well as challenges in a newly evolving era of lung cancer screening.
\end{abstract}

Recent findings-Lung cancer, seen in three-fold excess in HIV-infected populations, has become the most common non-AIDS defining malignancy in the highly active anti- retroviral therapy (HAART) era. HIV-associated lung cancer appears to be associated with young age at diagnosis, cigarette smoking, advanced stage at presentation, and a more aggressive clinical course. There is no unified explanation for these observations, and aside from traditional risk factors, HIV-related immunosuppression and biological differences might play a role. In addition to smoking cessation interventions, screening and early cancer detection in HIV-infected populations are of high clinical importance, although evidence supporting lung cancer screening in this particularly high-risk subset are currently lacking, as are prospective studies of lung cancer therapy.

Summary-There is an urgent need for prospective clinical trials in HIV-associated lung cancer to improve understanding of lung cancer pathogenesis and to optimize patient care. Several clinical trials are in progress to address questions in cancer biology, screening and treatment for this significant cause of mortality in persons with HIV infection.

\section{Keywords}

lung cancer; HIV; AIDS; smoking cessation

\section{Introduction}

Lung cancer is the most common non-AIDS defining malignancy in the highly active antiretroviral therapy (HAART) era and represents the leading cause of cancer-related death among people living with HIV [1]. In the United States, the risk of lung cancer in the persons with AIDS has remained elevated since the pre-HAART era, with relative risks during the periods 1980-1989, 1990-1995, and 1996-2002 of 2.5 (95\% CI: 1.9-3.3), 3.3 (95\% CI: 2.9-3.8), and 2.6 (95\% CI: 2.1-3.1), respectively [2]. Although the current burden of lung cancer in persons with HIV infection is low, with an estimated 1,597 cases (454 in persons with HIV-only, without AIDS) diagnosed in 34 U.S. states between 2004 and 2007

Corresponding Author: Missak Haigentz, Jr., MD, Associate Professor of Clinical Medicine, Albert Einstein College of Medicine/ Montefiore Medical Center, 111 East $210^{\text {th }}$ Street, Hofheimer Room 100, Bronx, New York 10467, Tel. 718.920.4826, mhaigent@montefiore.org. 
[3], this number is expected to increase with aging of the HIV-infected population.

Moreover, lung cancer in the setting of HIV infection is diagnosed nearly a decade and a half earlier in life than the general population. The reasons for this apparent age-inequality remain unclear, as are the risk factors predisposing to the development of this malignancy in the absence of known viral associations. In this review, we explore current questions regarding risk factors predisposing HIV-infected populations to the development of lung cancer at an early age, treatment options and outcomes of lung cancer therapy for HIVinfected individuals as well as current challenges in a newly evolving era of lung cancer screening.

\section{Question 1) What is responsible for the excess risk for lung cancer among persons with HIV infection?}

The aging of the HIV-positive population has been associated with increasing prevalences of several non-AIDS defining cancers (NADCs), including lung cancer [3, 4]. In the past, persons with HIV infection would likely have died of opportunistic infection or other diseases associated with severe immunodeficiency (e.g., lymphoma or Kaposi sarcoma) before they could develop lung cancer. Currently, lung cancer is seen in approximately three-fold excess among populations with HIV infection and is diagnosed at a comparatively young age, frequently in the fourth decade of life. An explanation for these observations may contribute to a better understanding of lung cancer pathogenesis and would likely impact the care and prevention of lung cancer in HIV-infected and uninfected populations alike.

\section{Smoking}

Cigarette smoking is the most important risk factor for developing lung cancer [5], and HIV positive individuals are known to have higher rates of tobacco use $[6,7,8]$. From a number of retrospective studies, it is evident that approximately half of HIV-infected individuals are smokers and tobacco exposure tends to start earlier and last longer than in the general population $[9,10]$. The prevalence of smoking among individuals with HIV infection ranges between 40 to $70 \%$, compared to about $20 \%$ in the general population [11, 12]. In several case series, nearly all patients with HIV-associated lung cancers were smokers [13*]. Recent data from the Veterans Aging Cohort Study-Virtual Cohort (VACS-VC) showed that current and former smokers with HIV infection had a substantially greater risk of lung cancer compared with never smokers (current smokers IRR: 6.3; 95\% CI: 4.7-8.4; former smokers IRR: 3.0, 95\% CI: 2.2-4.1) [14*].

While these statistics hold true for many retrospective studies, others indicate that the increased incidence of lung cancer is independent of smoking and alternative explanations are offered, including the role of immunosuppression $[15,16]$. Additionally, the reported excess in tobacco exposure among HIV-positive populations would suggest a preponderance of histological types of lung cancer more closely related to smoking (small cell and squamous cell histologies), which does not appear to be the case in studies reporting tumor histology $[15,17]$. Given tobacco's known role in lung carcinogenesis, smoking cessation recommendations and interventions should remain a critical part of routine clinical encounters in this high-risk population.

Earlier age at lung cancer diagnosis among persons with HIV infection remains incompletely understood from the available data, and smoking alone does not offer full explanation [18]. It is noted that the median time of diagnosis of lung cancer in HIV-positive individuals tends to occur in the middle of the fourth decade of life, about 15 years earlier than in the general population [13*]. Several prior studies suggested that increased rates of 
pulmonary infections in addition to altered lung function and enhanced lung damage among HIV-infected individuals might predispose these persons to lung cancer $[19,20]$. The impact of early age at cancer diagnosis has critical implications for cancer screening and opportunities for intervention (see below).

\section{Immunosuppression}

Immunosuppression might play an important role in the development of lung cancer. This concept was initially explored in the meta-analysis done by Grulich and colleagues in which the lung cancer incidence in organ transplantation recipients on immunosuppressive agents was comparable to that of persons with HIV infection [21]. This hypothesis was further supported by two large studies linking the development of AIDS to the increase in lung cancer incidence $[22,23]$.

Immunosuppression caused by HIV infection results in chronic immune activation, inflammation, immune system dysfunction and accelerated aging, all likely promoting carcinogenesis in this population [24, 25]. However, the relationship between CD4+ cell count, degree of immunosuppression and subsequent predisposition for lung cancer is not simple and remains unclear. A large, multi-centered cohort study of 20,775 HIV-infected persons showed a trend towards increased incidence of lung cancer in those with CD4+ count $\leq 200$ cells/ $\mu$ L (RR: 2.2; CI: 1.3-3.6) [26]. In contrast, a study by Prosperi and colleagues reported increased incidence of non-AIDS defining cancers (NADCs) in persons with CD4+ cell count $\geq 500$ cells/ $\mu \mathrm{L}$ [27]. While some studies indicate that the degree of immunodeficiency is associated with lung cancer risk [28, 29], others note that the duration of immunodeficiency with CD4+ cell count of $\leq 200$ cells $/ \mu \mathrm{L}$ might be more closely associated with this risk [30]. However, some studies have failed to demonstrate an association of low CD4+ cell count and the risk of lung cancer when adjusted for other risk factors [31, 15]. A cohort study of 37,294 HIV-infected persons showed no association of lung cancer with low CD4+ cell count, and moreover, an association with low baseline HIV RNA level was reported ( $<<0.01$ ); in this study, HIV infection was found to be independently associated with increased risk of lung cancer when adjusted for smoking and age [14*].

\section{Direct Viral Effect and Effects of Antiretroviral Therapies}

The discovery and advent of HAART corresponds to the period of time where increases in NADC rates, particularly for lung cancer, were noted, calling into question a possible role of antiretroviral agents in cancer risk. It has been suggested that nucleoside analogs with activity against HIV may cause host cell DNA damage and mutations that potentially impose a cancer risk [32]. Fortunately, in the few studies that have approached this question (all with relatively small numbers of HIV-infected persons), antiretroviral therapies have not been shown to increase lung cancer risk $[33,34,35]$.

Possible direct effects of HIV infection on carcinogenesis are poorly understood. HIV gene products, such as Tat protein, may play a role in promoting somatic mutations (Tat specifically inhibits DNA repair processes), potentially eventuating in carcinogenesis [36]. In a recent publication, expression of the HIV protein Nef was detected in 7 of 21 (33.3\%) head and neck squamous cell carcinomas in patients with HIV infection, the significance of which is unknown [37].

\section{Biological differences}

Given the current era of molecular diagnostics and targeted anticancer agents, it is critical to understand the biology of lung cancer, the most frequent cause of cancer death in the United States and increasingly important cause of mortality among persons with HIV. It is unknown 
at this time whether the increased risk and early onset of lung cancer in HIV-positive population could be secondary to unique biological differences which can have clear implications for both cancer epidemiology and treatment. For example, the recent discoveries of specific epidermal growth factor receptor (EGFR) mutations and ALK-EML4 gene rearrangements in small subsets of non-small cell lung cancer patients without extensive smoking histories have shed important light on lung cancer pathogenesis and revolutionized treatment approaches with specific therapies targeting the driver mutation $[38,39]$. An ongoing clinical trial by the NCI Genomics Program and the AIDS Malignancy Consortium (AMC-083; NCT01567722) will comprehensively sequence the tumor and normal genomes of cancer patients, including 100 persons with HIV-associated non-small cell lung cancer. This project will probe for oncogenic viral genomes, unique molecular differences, and distinctive immunologic factors in well-annotated clinical specimens. Understanding lung cancer biology in HIV infection is therefore expected to provide critical answers to questions regarding epidemiology and treatment (see below).

\section{Question 2) Do lung cancer patients with HIV infection have worse outcomes?}

Historically, it has been reported that HIV-positive individuals tend to have more advanced cancer stage at diagnosis that might contribute to poorer outcomes $[18,40]$. Whether it holds true nowadays is less clear with recent studies yielding contradictory findings. Silverberg and colleagues recently presented an analysis of incident cancer diagnoses over 1996-2009 from Surveillance Epidemiology and End Results (SEER)-reporting Kaiser Permanente cancer registries indicating 5-year survival rates of 7.9\% in 63 HIV-infected lung cancer patients compared to $21.9 \%$ in 394 uninfected lung cancer patients (HR death: 1.7; 95\% CI: $1.3-2.2 ; \mathrm{p}<0.001)$, with a trend toward more advanced staged cancers among patients with HIV-infection $(p=0.059)$ [41]. Several explanations have been proposed for poor outcomes, including immunosuppression, restricted access to medical care, inequality in treatment options and possibly more aggressive types of lung cancer [8, 42*]. The degree of immunosuppression seems to play a prognostic role, with low or declining CD4+ cell counts associated with worse outcomes and increased mortality [8, 40, 42*].

Another explanation for the observed poorer outcomes in HIV-infected lung cancer patients might be related to treatment disparities. In a recent study linking HIV and cancer registries in Texas, Suneja et al. [43*] found that HIV-infected patients were less likely to receive non-small cell lung cancer (NSCLC) treatment than uninfected cases (60.3\% vs. $77.5 \%$; odds ratio 0.39 ; $95 \%$ CI: 0.30-0.52). In this study, HIV-infected patients with localized NSCLC were less likely than their uninfected counterparts to undergo surgery, and those with metastatic disease were less likely to receive chemotherapy. Whether they were treated or not, HIV-infected patients had higher cancer-related mortality, suggesting that HIV infection itself plays an important role in cancer outcomes [43*].

However, the observations of poorer outcomes in HIV-associated lung cancer have recently been challenged. The Veterans Aging Cohort Study demonstrated that HIV-seropositive and HIV-uninfected individuals had similar lung cancer stages at diagnosis, with stages III and IV comprising the majority of the cases (68\% and 71\%, respectively) [14]. Another study based on the Surveillance Epidemiology and End Results (SEER) cancer registry from 2000 and 2005 evaluating 322 persons with HIV-associated non-small cell lung cancer compared to 71,976 HIV-negative controls with lung cancer showed no difference in stage at lung cancer diagnosis [44*]. Interestingly, early stage (I/II) NSCLC was diagnosed at higher frequency in the HIV-positive (35.4\%) versus HIV-negative population (27.8\%). The median survival between two groups for patients with early stage disease was similar. For stage IV disease, the median survival was 3 months for both groups $(\mathrm{p}=0.77)$. For stage III 
disease, however, survival appeared better in the HIV-negative cohort ( 7 vs. 3 months; $p=$ 0.051), but this difference was not statistically significant after adjustment for relevant covariates. Finally, the effect of treatment on mortality was examined for early stage disease between the two groups. HIV-infected patients who underwent surgical resection had similar survival when compared to their uninfected counterparts (50 months vs. 58 months, $\mathrm{p}=$ $0.88)$.

\section{Question 3) Should lung cancer patients with HIV receive different therapy?}

The choice of appropriate therapy for HIV-infected patients with lung cancer is a matter of uncertainty due to the lack of prospective and randomized trials in this population. HIVinfected individuals have been historically excluded from therapeutic cancer trials due to concern for toxicity, poor tolerability, and drug interactions with HAART [45, 46]. Therefore, most of the data on treatment outcomes come from the retrospective reviews or observational studies [42*]. Due to the lack of guidelines, treatment of HIV-positive individuals with lung cancer tends to vary based upon patient performance status and degree of immunosuppression, and the oncologist's experience.

In the absence of scientific evidence to support otherwise, it is generally recommended to treat HIV-positive patients with lung cancer similarly to uninfected patients. However, whether this recommendation holds true in real practice is unclear. Suneja and colleagues recently reported that treatment of HIV-infected individuals with non-small cell lung cancer (NSCLC) were less likely than HIV-uninfected individuals to receive surgery $(45.5 \%$ vs. $62.5 \%, \mathrm{p}=0.04)$. Particularly, the HIV-infected group was more frequently untreated for local stage NSCLC ( $41.9 \%$ vs. $14.4 \%$, $\mathrm{p}<0.0001)$, less often treated with chemotherapy or surgery for regional stage NSCLC, and less frequently treated with chemotherapy $31.1 \%$ vs. $45.5 \%$ ) for distant stage disease. The HIV-infected population was also more likely to receive no treatment $(43.4 \%$ vs. $27.4 \%, \mathrm{p}<0.0001)$ for advanced stage disease [43*].

Radiation therapy commonly used for locally advanced lung cancer has also been noted to have potentially increased toxicity in HIV-infected patients. A few older case series suggested that HIV-infected individuals had higher rates of radiation-related esophagitis and odynophagia [47, 48]; however these data come from very small patient samples and require additional validation.

A valid concern limiting systemic cancer therapy in persons with HIV receiving HAART medications is potential drug interactions with chemotherapeutic agents. Several cytotoxic agents frequently employed as lung cancer therapy (including the taxanes, vinca alkaloids and etoposide) are metabolized by cytochrome P450, and certain antiretroviral agents, especially protease inhibitors, inhibit cytochrome $\mathrm{P} 450$ enzymes, possibly potentiating the toxic effects of chemotherapeutic regimens [49]. A similar potential interaction exists between protease inhibitors and erlotinib or gefitinib in the treatment of lung cancer. However, little information on the clinical significance of these interactions in practice is currently available, and authoritative recommendations for dosing of antineoplastic agents in the setting of HAART do not exist.

Currently, two prospective studies are evaluating chemotherapeutic options specifically in HIV-infected populations. The Intergroupe Francophone de Cancerologie Thoracique has initiated a phase II trial of carboplatin plus pemetrexed in HIV-infected patients with advanced NSCLC (NCT01296113). In the United States, an AIDS Malignancy Consortium trial is investigating the carboplatin/paclitaxel regimen in HIV-infected patients with advanced solid tumors, including lung cancers (AMC-078, NCT01296113). The outcomes 
of these studies will provide a better understanding of treatment options, possible drug-drug interactions, and clinical outcomes.

A recent publication by Srirangam and colleagues has raised the intriguing possibility that ritonavir, a protease inhibitor often used in antiretroviral therapy, might be a potential therapeutic agent in the treatment of NSCLC by inhibiting survivin, an important antiapoptotic protein that is associated with chemotherapy resistance in NSCLC [50]. Nelfinavir, another protease inhibitor, has been studied as a potential radiosensitizer in lung cancer patients without HIV [51]. Thus, some antiretroviral agents may serve dual roles as both HIV and antineoplastic treatments.

\section{Question 4) Is there a role for lung cancer screening in HIV-positive populations?}

With the increased number of non-AIDS defining malignancies, cancer screening has become an important component of health maintenance in HIV clinical practice. Yet lung cancer is often diagnosed at late stages in HIV infected populations despite increased encounters with the medical system [52]. Although new guidelines for lung cancer screening in at risk populations have recently been developed by the National Comprehensive Cancer Network (NCCN) and the American Cancer Society (ACS) [53, 54], data supporting screening of HIV-infected populations are lacking.

The National Lung Screening Trial (NLST), which compared annual low-dose computed tomography (CT) vs. chest radiography for three consecutive years in persons with heavy smoking histories, confirmed benefits of screening for lung cancer in a high-risk population. This trial included 53,454 participants, age 55-74 with $>30$ pack-year history of smoking. A $20 \%$ relative reduction in lung cancer mortality and $7 \%$ relative reduction in all-cause mortality in participants screened with low-dose CT scans were observed [55], and as a result low-dose CT screening of persons meeting these criteria has been advocated.

Although the NLST has been a major advance in preventing lung cancer deaths, its results cannot necessarily be extrapolated to the HIV-infected population. The age for NLST trial eligibility was 55-74 years, an age range much older than the median age at lung cancer diagnosis in the setting of HIV infection. Additionally, most study participants were Caucasians (91\%), in contrast to the largely minority demographics of HIV-infection in the United States. Although the NLST did not exclude persons with HIV infection, it is unknown how many participants had HIV infection. As a result, it is unclear whether the findings of NLST are applicable to the HIV-infected population.

Furthermore, persons with HIV infection are at greater risk for infections and have nonspecific lymphadenopathy, which may increase the likelihood of false positive CT scans and unnecessary evaluations. To address this concern, Sigel et al. recently presented an evaluation of baseline chest CT scans from 145 HIV-positive and 125 uninfected veterans enrolled in the prospective, multicenter Examinations of HIV Associated Lung Emphysema (EXHALE) Study, a substudy of the Veterans Aging Cohort Study. The median age of the HIV-positive and uninfected study populations were 54 and 52, respectively, and the median CD4+ cell count among the HIV-infected subjects was 425 cells $/ \mu \mathrm{L}$. Although the frequency of abnormal pulmonary nodules was similar between the two groups (50\% vs. $46 \%$; $\mathrm{p}=$ $0.6)$, HIV-infected subjects tended to have more lymphadenopathy ( $15 \%$ vs. $7 \% ; \mathrm{p}=0.06)$. However, the median pack years of smoking history was low in both groups (HIV-positive: 26; Uninfected: 23), and this study population included never smokers (14\% and $18 \%$ of subjects, respectively) [56]. 
The French National Institute for Health and Medical Research-French National Agency for Research on AIDS and Viral Hepatitis (Inserm-ANRS) has initiated a prospective, multicenter study evaluating the prevalence of lung cancers detected by low-dose CT in 450 persons with HIV infection (HIV-CHEST, NCT01207986). Eligibility criteria for this study include age $\geq 40$ years, a smoking history $\geq 20$ pack-years (either active or with less than 3 years of cessation) and a nadir CD4+ count $<350$ cells $/ \mu$. Once completed, this study is expected to provide valuable information on the value of radiologic screening and stage at diagnosis in this high-risk population.

\section{Conclusions}

Lung cancer has become the most prevalent non-AIDS defining malignancy in the era of HAART. Increased prevalences of smoking, immunosuppression, pulmonary infection, and potential biological differences in persons with HIV all likely play roles in the early onset of lung cancer in this population. Effective, modern screening protocols hold promise as a means of limiting future mortality. However, there are no data from prospective randomized trials to support screening with low dose CT scans in this population. Effective smoking cessation interventions should certainly be emphasized, and may represent the most costeffective strategy to limit the future impact of lung cancers and other important tobaccorelated morbidities. Due to the exclusion of HIV-positive individuals from therapeutic lung cancer trials, optimal therapy remains unknown, although prospective trials are underway to answer this important question. A better understanding of the molecular biology of HIVassociated lung cancer may provide insight into current epidemiological questions and permit targeted cancer therapy. Additional research is needed, ideally from prospective studies, to better elucidate the epidemiology, pathogenesis, and optimal diagnostic, treatment, and prevention approaches for lung cancer in those living with HIV in order to optimize the medical response to this significant health challenge.

\section{Acknowledgments}

This work was funded by the Clinical Core of the Center for AIDS Research at the Albert Einstein College of Medicine and Montefiore Medical Center (NIH AI-51519) and the AIDS Malignancy Consortium Grant (U01CA121947).

\section{References}

1. Simard EP, Pfeiffer RM, Engels EA. Cumulative incidence of cancer among individuals with acquired immunodeficiency syndrome in the United States. Cancer. 2011; 117:1089-1096. [PubMed: 20960504]

2. Engels EA, Pfeiffer RM, Goedert JJ, et al. Trends in cancer risk among people with AIDS in the United States 1980-2002. AIDS. 2006; 20:1645-1654. [PubMed: 16868446]

3. Shiels MS, Pfeiffer RM, Gail MH, et al. Cancer burden in the HIV-infected population in the United States. J Natl Cancer Inst. 2011; 103:753-762. [PubMed: 21483021]

4. Pantanowitz L, Dezube BJ. Evolving spectrum and incidence of non-AIDS-defining malignancies. Curr Opin HIV AIDS. 2009; 4:27-34. [PubMed: 19339937]

5. Flanders WD, Lally CA, Zhu BP, et al. Lung cancer mortality in relation to age, duration of smoking, and daily cigarette consumption: results from Cancer Prevention Study II. Cancer Res. 2003; 63:6556-6562. [PubMed: 14559851]

6. Spano JP, Massiani MA, Bentata M, et al. Lung cancer in patients with HIV infection and review of the literature. Med Oncol. 2004; 21:109-115. [PubMed: 15299182]

7. Ruiz M. Lung Cancer in HIV-infected patients: the experience of an urban clinic. J Int Assoc Physicians AIDS Care (Chic Ill). 2010; 9:214-217. 
8. Brock MV, Hooker CM, Engels EA, et al. Delayed diagnosis and elevated mortality in an urban population with HIV and lung cancer: implications for patient care. J Acquir Immune Defic Syndr. 2006; 43:47-55. [PubMed: 16936558]

9. Giordano TP, Kramer JR. Does HIV infection independently increase the incidence of lung cancer? Clin Infect Dis. 2005; 40:490-491. [PubMed: 15668878]

10. Shiels MS, Pfeiffer RM, Engels EA. Age at cancer diagnosis among persons with AIDS in the United States. Ann Intern Med. 2010; 153:452-460. [PubMed: 20921544]

11. Burkhalter JE, Springer CM, Chhabra R, et al. Tobacco use and readiness to quit smoking in lowincome HIV-infected persons. Nicotine and Tobacco Research. 2005; 7:511-522. [PubMed: 16085522]

12. Centers for Disease Control and Prevention. Vital Signs: Current Cigarette Smoking Among Adults Aged 18 Years-United States, 2009. Morbidity and Mortality Weekly Report. 2010; 59:1135-1140. [PubMed: 20829747]

13. Mani D, Haigentz M Jr, Aboulafia DM. Lung cancer in HIV Infection. Clin Lung Cancer. 2012; 13:6-13. [PubMed: 21802373] . A comprehensive review article on risk factors, screening, treatment and outcomes in HIV-associated lung cancer.

14. Sigel K, Wisnivesky J, Gordon K, et al. HIV as an independent risk factor for incident lung cancer. AIDS. 2012; 26:1017-1025. [PubMed: 22382152] . This article presents data from the Veterans Aging Cohort Study Virtual Cohort (VACS-VC) that showed that the presence of HIV infection appears a risk factor for lung cancer even when controlling for many other variables.

15. Kirk GD, Merlo C, O'Driscoll P, et al. HIV infection is associated with an increased risk for lung cancer, independent of smoking. Clin Infect Dis. 2007; 45:103-110. [PubMed: 17554710]

16. Engels EA, Brock MV, Chen J, et al. Elevated incidence of lung cancer among HIV infected individuals. J Clin Oncol. 2006; 24:1383-1388. [PubMed: 16549832]

17. Jung G, Chung J, Shuter J, et al. Lung Cancer in HIV Infection: A case series. J Clin Oncol. 2008; 26(suppl) (abstr 19093).

18. Chaturvedi AK, Pfeiffer RM, Chang L, et al. Elevated risk of lung cancer among people with AIDS. AIDS. 2007; 21:207-213. [PubMed: 17197812]

19. Pacht ER, Diaz P, Clanton T, et al. Alveolar fluid glutathione decreases in asymptomatic HIVseropositive subjects over time. Chest. 1997; 112:785-788. [PubMed: 9315816]

20. Littman AJ, Jackson LA, Vaughan TL. Chlamydia pneumonia and lung cancer: epidemiologic evidence. Cancer Epidemiol Biomarkers Prev. 2005; 14:773-778. [PubMed: 15824142]

21. Grulich AE, van Leeuwen MT, Falster MO, et al. Incidence of cancers in people with HIV/AIDS compared with immunosuppressed transplant recipients: a meta-analysis. Lancet. 2007; 370:5967. [PubMed: 17617273]

22. Shiels MS, Cole SR, Kirk GD, et al. A meta-analysis of the incidence of non-AIDS cancers in HIV-infected individuals. J Acquir Immune Defic Syndr. 2009; 52:611-622. [PubMed: 19770804]

23. Engels EA, Pfeiffer RM, Goedert JJ, et al. Trends in cancer risk among people with AIDS in the United States 1980-2002. AIDS. 2006; 20:1645-1654. [PubMed: 16868446]

24. Grivennikov SI, Greten FR, Karin M. Immunity, inflammation, and cancer. Cell. 2010; 140:883899. [PubMed: 20303878]

25. Pine SR, Mechanic LE, Enewold L, et al. Increased levels of circulating interleukin 6, interleukin 8, C-reactive protein, and risk of lung cancer. J Natl Cancer Inst. 2011; 103:1112-1122. [PubMed: 21685357]

26. Silverberg MJ, Chao C, Leyden WA, et al. HIV infection, immunodeficiency, viral replication, and the risk of cancer. Cancer Epidemiol Biomarkers Prev. 2011; 20:2551-2559. [PubMed: 22109347]

27. Prosperi MCF, Cozzi-Lepri A, Castagna A, et al. Icona Foundation Study Group. Incidence of malignancies in HIV-infected patients and prognostic role of current CD4 cell count: evidence from a large Italian cohort study. Clin Infect Dis. 2010; 50:1316-1321. [PubMed: 20297953]

28. Guiguet M, Boue F, Cadranel J, et al. Clinical Epidemiology Group of the FHDHANRSCO4 Cohort. Effect of immunodeficiency, HIV viral load, and antiretroviral therapy on the risk of individual malignancies (FHDH-ANRS CO4): a prospective cohort study. Lancet Oncol. 2009; 10:1152-1159. [PubMed: 19818686] 
29. Reekie J, Kosa C, Engsig F, et al. Relationship between current level of immunodeficiency and nonacquired immunodeficiency syndrome-defining malignancies. Cancer. 2010; 116:5306-5315. [PubMed: 20661911]

30. Kesselring A, Gras L, Smit C, et al. Immunodeficiency as a risk factor for non-AIDS-defining malignancies in HIV-1-infected patients receiving combination antiretroviral therapy. Clin Infect Dis. 2011; 52:1458-1465. [PubMed: 21628488]

31. Clifford GM, Polesel J, Rickenbach M, et al. Cancer risk in the Swiss HIV Cohort Study: associations with immunodeficiency, smoking, and highly active antiretroviral therapy. J Natl Cancer Inst. 2005; 97:425-432. [PubMed: 15770006]

32. Carter MM, Torres SM, Cook DL Jr, et al. Relative mutagenic potencies of several nucleoside analogs, alone or in drug pairs, at the HPRT and TK loci of human TK6 lymphoblastoid cells. Environ Mol Mutagen. 2007; 48:239-247. [PubMed: 17358029]

33. Powles T, Robinson D, Stebbing J, et al. Highly active antiretroviral therapy and the incidence of non-AIDS-defining cancers in people with HIV infection. J Clin Oncol. 2009; 27:884-890. [PubMed: 19114688]

34. Krishnan S, Schouten JT, Jacobson DL, et al. Incidence of non-AIDS-defining cancer in antiretroviral treatment-naïve subjects after antiretroviral treatment initiation: an ACTG longitudinal linked randomized trials analysis. Oncology. 2011; 80:42-49. [PubMed: 21606663]

35. Chao C, Leyden WA, Xu L, et al. Exposure to antiretroviral therapy and risk of cancer in HIVinfected persons. AIDS. 2012; 26:2223-2231. [PubMed: 22951631]

36. Nunnari G, Smith JA, Daniel R. HIV-1 Tat and AIDS-associated cancer: targeting the cellular anticancer barrier? J Expr Clin Cancer Res. 2008 May 15.27(1):3.

37. McLemore MS, Haigentz M Jr, Smith RV, et al. Head and neck squamous cell carcinomas in HIVpositive patients: a preliminary investigation of viral associations. Head Neck Pathol. 2010 Jun; 4(2):97-105. [PubMed: 20333562]

38. Maemondo M, Inoue A, Kobayashi K, et al. Gefitinib or chemotherapy for non-small-cell lung cancer with mutated EGFR. N Engl J Med. 2010; 362:2380-2388. [PubMed: 20573926]

39. Vadakara J, Borghaei H. Personalized medicine and treatment approaches in non- small-cell lung carcinoma. Pharmgenomics Pers Med. 2012; 5:113-123. [PubMed: 23226067]

40. Lavole A, Wislez M, Antoine M, et al. Lung cancer, a new challenge in the HIV-infected population. Lung Cancer. 2005; 51:1-11. [PubMed: 16300854]

41. Silverberg, M.; Chao, C.; Leyden, W., et al. Cancer stage, age at diagnosis, and survival comparing $\mathrm{HIV}^{+}$and $\mathrm{HIV}^{-}$individuals with common non-AIDS-defining cancers; 2012 CROI Meeting; Abstract \#903.

42. Pakkala S, Chen Z, Rimland D, et al. Human immunodeficiency virus-associated lung cancer in the era of highly active antiretroviral therapy. Cancer. 2012; 118:164-172. [PubMed: 21713759] . This retrospective review of 80 patients with HIV-associated lung cancer at a single institution emphasized an observation that higher CD4+ cell count was associated with improved outcomes.

43. Suneja G, Shiels MS, Melville SK, et al. Disparities in the treatment and outcomes of lung cancer among HIV-infected people in Texas. AIDS. 2013; 27:459-468. [PubMed: 23079809] . This cancer and HIV-linked, registry-based study highlights differences in treatment and outcomes in HIV infected patients and reports less favorable outcomes and lack of appropriate and aggressive treatment in this population.

44. Rengan R, Mitra N, Liao K, et al. Effect of HIV on survival in patients with non-small-cell lung cancer in the era of highly active antiretroviral therapy: a population-based study. Lancet Oncol. 2012; 13:1203-1209. [PubMed: 23164952] . This SEER-based study did not identify significant differences in clinical outcomes between patients with HIV and uninfected patients with non-small cell lung cancer.

45. Persad GC, Little RF, Grady C. Including persons with HIV infection in cancer clinical trials. J Clin Oncol. 2008; 26:1027-1032. [PubMed: 18309938]

46. Weiss SA, Gajavelli S, Negassa A, et al. Evaluation of cancer trial eligibility criteria (with focus on non-AIDS-defining cancers) for inclusion of persons with HIV infection. J Clin Oncol. 2011; 29(suppl) (abstr 6092). 
47. Tirelli U, Spina M, Sandri S, et al. The Italian Cooperative Group on AIDS and Tumors. Lung carcinoma in 36 patients with human immunodeficiency virus infection. Cancer. 2000; 88:563569. [PubMed: 10649248]

48. Leigh BR, Lau DH. Severe esophageal toxicity after thoracic radiation therapy for lung cancer associated with the human immunodeficiency virus: a case report and review of the literature. Am J Clin Oncol. 1998; 21:479-481. [PubMed: 9781604]

49. Rudek MA, Flexner C, Ambinder RF. Use of antineoplastic agents in cancer patients with HIV/ AIDS. Lancet Oncol. 2011; 12:905-912. [PubMed: 21570912]

50. Srirangam A, Milani M, Mitra R, et al. The HIV protease inhibitor ritonavir inhibits lung cancer cells, in part, by inhibition of survivin. J Thorac Oncol. 2011; 6:661-670. [PubMed: 21270666]

51. Rengan R, Mick R, Pryma D, et al. A phase I trial of the HIV protease inhibitor nelfinavir with concurrent chemoradiotherapy for unresectable stage IIIA/IIIB non- small cell lung cancer: a report of toxicities and clinical response. J Thorac Oncol. 2012; 7:709-715. [PubMed: 22425919]

52. Bedimo RJ, McGinnis KA, Dunlap M, et al. Incidence of non-AIDS-defining malignancies in HIV-infected versus noninfected patients in the HAART era: impact of immunosuppression. J Acquir Immune Defic Syndr. 2009; 52:203-208. [PubMed: 19617846]

53. National Comprehensive Cancer Network. [Accessed on April 8, 2013] Lung Cancer screening. 2013. http://www.nccn.com/files/cancer-guidelines/lung_screening/index.html

54. American Cancer Society. [Accessed on April 8, 2013] Non-small cell lung cancer detection. 2013. http://www.cancer.org/cancer/lungcancer-non-smallcell/detailedguide/non-small-cell-lung-cancerdetection

55. Aberle DR, Adams AM, Berg CD, et al. National Lung Screening Trial Research Team. Reduced lung-cancer mortality with low-dose computed tomographic screening. N Engl J Med. 2011; 365:395-409. [PubMed: 21714641]

56. Sigel, M.; Brown, S.; Wisnivesky, J., et al. Chest CT scan findings and implications for lung cancer screening in asymptomatic HIV infected patients; 2012 CROI Meeting Abstract \#907; 


\section{Key Points}

- Lung cancer, the leading cause of cancer death in persons with HIV during the HAART era, is diagnosed approximately three-fold more frequently and presents nearly a decade and a half earlier in life among persons with HIV infection.

- Although cigarette smoking remains the greatest target for increased lung cancer risk in HIV infection, HIV-associated immunosuppression and as yet undiscovered biological factors are also likely involved.

- The historical observations of poorer lung cancer outcomes in HIV infection have been recently challenged and may be due, at least in part, to inadequate cancer therapy.

- Given limitations of scientific evidence derived from current case series reports and frequent exclusion of persons with HIV infection from lung cancer trials, prospective studies incorporating translational research are urgently needed to address important questions in lung cancer biology, screening, therapy and outcomes in this currently underserved population. 